\title{
2017 Reviewer Acknowledgement
}

\section{Editorial Office of Journal of Exploratory Research in Pharmacology}

We thank the following reviewers for their contribution and support in 2017.

\author{
Kandru Ammani \\ India \\ Alessandra Arcolaci \\ Italy \\ Muniappan Ayyanar \\ India \\ Sidhanath V. Bhosale \\ India \\ Willian O. Castillo \\ Colombia \\ Manab Chakravarty \\ India \\ Chang Chen \\ United States \\ Heyu Chen \\ United States \\ Luciene Ferreira de Lima \\ Brazil \\ M De Rosa \\ Italy \\ Murali Dhanasekaran \\ United States \\ Jianxun Ding \\ China \\ Salah Mohamed EI Sayed \\ Egypt \\ Iriana Galán-Arriero \\ Spain \\ Upendra C. Galgatte \\ India \\ Raghu Ganugula \\ United States
}

\author{
Ankur Gogoi \\ India \\ Xinsheng Gu \\ China \\ Surampalli Gurunath \\ India \\ G. Hervás \\ Spain \\ Nasir M. Idkaidek \\ Jordan \\ Robbert J. Kok \\ Netherlands \\ Agbaje Lateef \\ Nigeria \\ Glen Lawrence \\ United States \\ Junxia Li \\ China \\ P. P. Mathur \\ India \\ Ahmed E. Abdel Moneim \\ Egypt \\ Kesara Na-Bangchang \\ Thailand \\ Khue Vu Nguyen \\ United States \\ Anna Pratima G. Nikalje \\ India \\ Hilal Özdağ \\ Turkey \\ Alice L. Perez \\ Costa Rica
}

\section{Claudio Pirozzi}

Italy

Rajendran Amarnath Praphakar India

Ying Qu

United States

D. Rapaka

India

Celestino Sardu

Italy

Radhika Sharma

India

Miroslav Simunic Croatia

Rajesh K. Singh India

Eleonora Stanca Italy

Norman Stockbridge United States

Srijayaprakash Uppada United States

Jie Wang China

Yufeng Xie Canada

Xunyan Ye United States

Xiaojuan Zhang United States

Yanling Zhao China

DOI: 10.14218/JERP.2017.000RA 